\title{
Discurso y textos académicos. Definiciones para iniciar un centro de escritura normalista
}

\section{Academic texts and discourse. Definitions to start a writing center in a teachers training school}

\author{
EVA MARGARITA GodínEz LóPEZ*
}

Este artículo revisa conceptos relacionados con la escritura de textos académicos desde una mirada funcionalista e interaccionista del discurso y el lenguaje, con el propósito de reflexionar sobre el objeto de los centros de escritura y establecer un marco teórico que fundamente la práctica de un centro dirigido a una comunidad normalista. La literacidad y la alfabetización académica se inscriben aquí en el contexto de la formación inicial de profesores. Se abordan los aspectos sociales y epistemológicos de las prácticas de escritura comunes en la educación superior, así como los lingüísticos que caracterizan los textos que median dichas prácticas y que pueden representar retos para los estudiantes. Se concluye que la razón práctica del centro de escritura ha de sustentarse en un posicionamiento teórico-pedagógico claro, basado en el conocimiento de las características del discurso y el lenguaje que se emplea en un proceso de inculturación que tiene un carácter social, cognoscitivo y lingüístico.

This paper examines various concepts related to academic texts writing from a functionalist and interactionist perspective of discourse and language, with the aim of reflecting on the purpose of writing centers and to establish a theoretical framework which substantiates the practice of a center aimed at teacher's education. In this work, literacy and academic literacy are circumscribed in the context of initial teachers training. Social and epistemological aspects of common writing practices in higher education are addressed, as well as the linguistic aspects that characterize these types of texts and that can represent challenges for students. It is concluded that the practical reason of the writing center must be grounded on a clear theoretical-pedagogical position, based on the knowledge of the features of discourse and language used in such process of inculturation, which has a social, cognitive and linguistic character.

\author{
Palabras clave: \\ literacidad, \\ literacidad \\ académica, \\ discurso \\ académico, \\ escritura \\ académica, \\ centros de escritura, \\ formación inicial \\ de profesores
}

\section{Keywords:}

literacy, academic literacy, academic discourse, academic writing, writing centers, initial teachers training.

Recibido: 28 de junio de 2020 | Aceptado para su publicación: 12 de enero de 2021 | Publicado: 12 de marzo de 2021

Recuperado de: https://sinectica.iteso.mx/index.php/SINECTICA/article/view/1172 doi: 10.31391/S2007-7033(2021)0056-010

\footnotetext{
* Doctora en Lingüística por la Facultad de Lenguas y Letras de la Universidad Autónoma de Querétaro y ex becaria del Consejo Nacional de Ciencia y Tecnología. Profesora-investigadora y miembro de la Comisión de Titulación en la Escuela Normal Superior Oficial de Guanajuato. Cofundadora del Centro de Recursos para la Escritura Académica Normalista en la Escuela Normal Oficial de Irapuato y coordinadora del Comité Editorial de las Escuelas Normales Públicas del Estado de Guanajuato. Líneas de investigación: escritura académica y procesos formativos. Correo electrónico: em.godinezlopez@ ensog.com.mx/https://orcid.org/0000-0002-8548-9321
} 


\section{INTRODUCCIÓN}

E

$n$ uno de los estudios que sentaron las bases de la alfabetización académica en Latinoamérica (Carlino, 2004, 2012) se hizo evidente que los centros o programas de escritura pioneros han abordado las características organizativas y las necesidades de las instituciones que los alojan antes de poner en práctica su programa. Este análisis contextual tiene sentido porque, como lo señalan varios autores (Halliday, 2007; Motta-Roth, 2009; Swales, 2013), desde una perspectiva funcional del lenguaje, alfabetizarse significa introducirse en una esfera de actividad y en una cultura en la cual las interacciones son mediadas por textos que, a su vez, son determinados por las situaciones que le atañen y contribuyen a configurarla como tal.

De este modo, la iniciativa de instalar un centro o un programa de escritura académica debe surgir, se supone, del conocimiento de esas situaciones, de las expectativas de la institución y los géneros que posibilitan y cristalizan dichas interacciones, todo lo cual permitiría perfilar el tipo de literacidad que esa cultura exigiría de los estudiantes y las acciones de alfabetización que se podrían promover.

Ese conocimiento habría de servir para tomar una postura pedagógica desde la cual se derive la actuación práctica de un centro; esto significa que, teniendo a la vista un desideratum -fruto de ese análisis situado-, las personas que emprenden una iniciativa de alfabetización académica incorporan el saber sobre los procesos de aprendizaje e inculturación disciplinar desde alguna perspectiva psicológica, antropológica, sociológica y lingüística, en la que se insertan nociones fundamentales como poder epistémico, proceso de composición, situación retórica, movida, sistema de actividad, práctica, práctica letrada, cultura letrada, acto de habla, elección lingüística o género, entre muchas otras, que al final se decantan en una "filosofía" institucional.

Thaiss et al. (2012) y Molina-Natera (2019b) revelan que las iniciativas universitarias han ido adoptando el modelo de alfabetización académica que les parece más adecuado a partir del conocimiento de otros centros o programas, si no es que han sido ellas mismas generadoras de conocimiento. En este sentido, en nuestro trabajo planteamos que, aunque no se puede saber con certeza en cuántos casos la creación de centros o programas de escritura académica es fruto de un análisis preciso del contexto institucional propio, sus particularidades y los retos que en materia de literacidad ofrece al alumnado, la apropiación de un marco teórico más o menos definido o, incluso, la adopción de tal o cual "filosofía" o plan programático, sí podemos afirmar la necesidad de un ejercicio analítico y de reflexión que fundamente el actuar pedagógico.

Molina-Natera (2019) sugiere que "gran parte de la información y el conocimiento sobre centros y programas de escritura se ha generado en Estados Unidos, lo cual implica un referente alejado del contexto regional tanto en lo cultural como en lo epistemológico" (p. 8), lo que podría dificultar este ejercicio de deliberación conceptual. No obstante, una de las recomendaciones que ofrecen los miembros de centros veteranos a los emergentes es que la misión y el posicionamiento teórico de este tipo de iniciativas se definan con el mayor rigor y claridad posibles, pues tales principios facilitan una actuación adecuada al interior de la institución, en tanto se dirige de modo asertivo a la población meta (Galán y Ormsby, 2011; Molina-Natera, 2014, 2015, 2016; Montes y Vidal, 2017; Moyano, 2017). 
Por ello, quienes fundamos el Centro de Recursos para la Escritura Académica Normalista nos propusimos hacer una reflexión sobre el objeto de los centros y programas de escritura en general para establecer un marco teórico-conceptual para la práctica de la alfabetización académica en un contexto específico: una institución formadora de docentes de educación básica.

Para cumplir ese propósito, hicimos una revisión profunda de la teoría sobre discurso especializado, académico, pedagógico y profesional, sus géneros y los textos que lo concretan en el ámbito escolar, y sistematizamos conceptos que están en la base de los movimientos contemporáneos de alfabetización académica para poder seleccionar los que resultaran acordes con las necesidades y los objetivos de una institución formadora de docentes. La revisión teórica se guio por las preguntas que Parodi (2007, 2018) y Navarro y Brown (2014) proponen para analizar funcionalmente el discurso: ¿dónde se produce y dónde circula el discurso?, ¿quién lo produce, para quién, para conseguir qué? y ¿qué opciones del sistema de la lengua realiza ese discurso?

Estas preguntas delimitan tres criterios: uno social, que da cuenta de los fines de los actos discursivos entre sujetos en un contexto dado; uno epistemológico e interactivo, que considera los tipos de conocimiento que son producidos y validados por medio del discurso en esos contextos y por esos sujetos interactuantes; y uno lingüístico, que ayuda a caracterizar y situar los recursos del sistema de la lengua que particularizan los textos que fungen como artefactos en esas interacciones discursivas contextualizadas.

En los siguientes apartados desarrollamos esta revisión conceptual, y en una sección final presentamos algunas conclusiones para el quehacer práctico del centro de escritura normalista.

\section{LITERACIDAD ACADÉMICA Y ALFABETIZACIÓN}

El concepto de literacidad ha sido definido de diversas maneras. Street $(1984,2005)$ explica que el concepto se ha enfocado de cuatro modos: la literacidad como aprendizaje, que la asimila a la alfabetización básica llevada a cabo en la infancia; los enfoques cognitivos, a los que subyace la idea de "la gran división" entre (pueblos e individuos) letrados y no letrados, asociada a los procesos de inculturación escolar basados en la escritura; el enfoque de los nuevos estudios de la literacidad, fundado en el concepto de práctica social, que considera las prácticas letradas como parte de contextos sociales y culturales, situadas históricamente e imbuidas por relaciones de poder (Barton \& Hamilton, 2010); y la perspectiva de la multimodalidad, que postula la existencia de literacidades múltiples, un repertorio de prácticas dentro de las cuales quedarían incluidas las prácticas escritas convencionales y emergentes (Anstey \& Bull, 2018; Cassany, 2012; Unesco, 2014).

Dada esta variedad de perspectivas, para operacionalizar el tipo de prácticas y de literacidad que buscamos promover a través de un centro de escritura, resulta útil considerar la definición de la Organización de las Naciones Unidas para la Educación, la Ciencia y la Cultura (Unesco):

The ability to identify, understand, interpret, create, communicate and compute, using printed and written materials associated what varying contexts. Literacy involves a continuum of learning in enabling individuals to achieve their goals, to develop their 
knowledge and potential, and to participate fully in their community and wider society (citado en Montoya, 2018).

Desde este punto de vista, literacidad es la habilidad para realizar un conjunto de prácticas letradas articuladas entre sí que pueden asociarse a un contexto institucional específico, como la familia, la escuela, el trabajo, la iglesia, la comunidad, entre otros (Zavala, 2009). Los conceptos de evento letrado y práctica letrada provienen del enfoque de los nuevos estudios de la literacidad (Barton \& Hamilton, 2010; Street, 2005). Se llama evento letrado cualquier ocasión en que las interacciones de los participantes involucran materiales escritos y, por lo tanto, implican procesos de interpretación de estos; dichos procesos son repetitivos e incluso ritualizados, como sucede en cualquier práctica institucional.

Por su parte, las prácticas letradas se centran en el entendimiento de estas interacciones como actos sociales situados y cargados de ideologías, es decir, de concepciones sobre el papel de la escritura, sus efectos, sus metas, su valor o prestigio, por lo cual algunas prácticas resultan más visibles e influyentes que otras (Barton \& Hamilton, 2010), algunas han sido dominantes y otras no, unas son percibidas como formales y otras como informales o vernáculas (Cassany y López-Ferrero, 2010).

La definición de la Unesco entra, así, en concordancia con la perspectiva de los nuevos estudios de la literacidad al asumir que las prácticas letradas escolares se inscriben dentro de un gran espectro en que se ven involucrados la escritura y los textos (convencionales y multimodales), en diferentes contextos sociales, y que dichas prácticas son realizadas por comunidades vinculadas por objetivos comunes.

Por su parte, la Organización para la Cooperación y el Desarrollo Económicos, en la operación de un programa de seguimiento a las habilidades de adultos, anota en este mismo concepto las habilidades para entender, evaluar, usar e involucrarse con textos para participar en la sociedad, lograr objetivos propios y desarrollar el propio conocimiento y potencial (Montoya, 2018).

De lo anterior se desprende que, en el contexto escolar, se llama literacidad escolar o académica a la capacidad de los estudiantes para adaptarse a las diversas situaciones en que se usa el lenguaje especializado que caracteriza los procesos de formación, y a las prácticas mediadas por ese lenguaje; mientras, la alfabetización académica sería el conjunto de acciones o esfuerzos por atender esa necesidad de adaptación dentro de las instituciones escolares (Carlino, 2003).

Según Bazerman (2005), literacidad académica es un término que combina la lectura y la escritura, pues estas nunca ocurren por separado; siempre son parte de un mismo campo de actividad; además, este tipo de literacidad se tiene que entender como una competencia transversal al currículo, ya que cada materia curricular tiene un lenguaje propio y en cada una se habla, se lee y se escribe para aprender (Álvarez, 2010). Esta sería, en consecuencia, la materia de la alfabetización académica.

Ahora bien, ¿qué tipo de literacidad corresponde a la educación superior? De acuerdo con lo señalado por Russell (1991), de que cada nivel y organismo educativo desarrolla su propia literacidad, Bazerman (2005) distingue dos procesos de inculturación de las prácticas letradas dependiendo de los propósitos de cada etapa de la formación académica. Por un lado, una suerte de "iniciación a la escolaridad": 
The term academic literacy is most widely used in reference to the lower and middle grades of schooling, to distinguish the kinds of reading and writing students are expected to do in school from the kinds of reading and writing children might do in their daily life outside of school (Bazerman, 2005, p. 7, cursivas añadidas).

Por otro lado, se refiere a un complejo proceso de inserción a la vida académica como puerta de acceso al campo profesional:

Academic language socialization is the process by which individuals learn to enter into the discussions and gain access to the resources of academic disciplines through learning specialized language use and participating in academic activity settings. Learning to read and write in academic settings occurs through extended experiences in those settings, by meeting the expectations of those situations, and gaining from the opportunities for participation they offer (Bazerman, 2005, p. 8, cursivas añadidas).

Este segundo proceso es lo que Parodi (2008) llama alfabetización disciplinar o especializada, en un medio en que los géneros escritos constituyen "accesos al conocimiento y a las prácticas especializadas escritas" (p. 31). En el marco de un centro de escritura, todas estas consideraciones han de tomarse en cuenta para comprender tanto el tipo de prácticas que ayudan al estudiante a iniciarse en la formación académica como la naturaleza misma de este proceso de inculturación.

De este modo, el término de literacidad académica atañe al sentido expresado en la primera cita de Bazerman (2005, p. 7), mientras que el de alfabetización escolar o académica, en cuyo interior se encuentra la competencia en escritura (Álvarez, 2010), se relaciona con la segunda (Bazerman, 2005, p. 8). Para entender la necesidad de estas precisiones terminológicas, en seguida caracterizamos el discurso y los textos en el ámbito escolar en relación con el tipo particular de habilidades cuyo dominio se intenta promover.

\section{EL DISCURSO EN EL CONTEXTO SOCIAL}

Desde una perspectiva funcionalista, el lenguaje es entendido como una herramienta semiótica que se usa para conseguir determinados fines en contextos sociales (Halliday, 2013). Las teorías funcionalistas "sostienen que la naturaleza del lenguaje se explica a partir de los objetivos sociales que guían su uso [...] son teorías interesadas por entender las relaciones entre los contextos socioculturales y situacionales del uso lingüístico y las características propias del lenguaje" (Navarro y Brown, 2014, p. 55).

El funcionalismo postula que la interacción entre lenguaje y contexto construye significados dentro del sistema de una lengua; por ello, se enfoca en las opciones lingüísticas sistemáticamente disponibles para los hablantes en diferentes contextos de uso. De este enfoque teórico se desprende que los enunciados orales y escritos reflejan las esferas comunicativas donde estos se producen, y que, a la vez, las opciones lingüísticas disponibles en el sistema ayudan a configurar esos contextos.

Considerada en este marco, la escuela es un contexto institucional donde los estudiantes se familiarizan con formas de usar el lenguaje diferentes a las que ya conocen, $y$, concomitantemente a esta inculturación, aprenden nuevos modos de pensar. En ese ámbito formal, los alumnos estudian los contenidos escolares de manera imbricada en el discurso, por medio de los géneros que contribuyen a construirlos; los 
miembros de la institución esperan de ellos que lleguen a dominar, a su vez, algunos de esos géneros, y los evalúan a partir de cómo expresan sus aprendizajes a través del lenguaje. Por ello, podemos decir que la escolarización es un proceso ante todo sociolingüístico (Schleppegrell, 2004).

Con base en este posicionamiento sociocognitivo, interaccionista y funcional ante el discurso, consideramos que entre todas las prácticas sociales que ocurren en las comunidades humanas existe un subtipo que se caracteriza por el uso situado y particular de ciertos "artefactos comunicativos letrados" (discursos cristalizados en textos) que las personas utilizan para conseguir objetivos relevantes en sus vidas. Estos artefactos son el resultado de una práctica histórica y cultural situada en cada comunidad. El manejo de esos artefactos letrados y de las prácticas letradas en que estos se usan se relaciona con la construcción de identidades sociales y con el ejercicio del poder al interior de esas comunidades (Cassany y López-Ferrero, 2010).

Las prácticas de escritura son múltiples y tienen lugar en cualquier grupo social que comparta propósitos comunicativos específicos. Una comunidad específica en la que se realizan prácticas letradas es la academia, donde se configuran diversas culturas disciplinares (Navarro y Revel, 2013). Aunque el ámbito académico ha sido concebido tradicionalmente como el contexto característico de aprendizaje, en el que se desarrollan prácticas letradas que, de modo usual, van sofisticándose y especializándose conforme se incrementa la complejidad del conocimiento, no es el único espacio donde se llevan a cabo; de hecho, en casi todos los ámbitos de la vida hay actividades que son mediadas por prácticas de lectura y escritura.

Existen manifestaciones vernáculas de prácticas letradas, más asociadas a los espacios personal, familiar y cultural donde se desenvuelven las vidas de las personas; estas son privadas y autogestionadas a diferencia de las prácticas académicas, que adquieren un carácter dominante y son estandarizadas y reguladas por las instituciones educativas (Cassany y López-Ferrero, 2010). A pesar de esta diversidad, las concepciones dominantes vinculadas a la academia, acerca de lo que significa leer y escribir, están tan interiorizadas que no es fácil "despedagogizarlas" (Street, 1995).

\section{DISCURSO ACADÉMICO Y DISCURSO PROFESIONAL}

Atendiendo el carácter situado de los usos del lenguaje y la escritura, diversos autores han identificado distintos tipos de discurso: académico, profesional, especializado o científico, entre otros atributos. El discurso académico es el lenguaje y las prácticas de discurso que ocurren en los centros de investigación, las universidades, las escuelas y en cualquier contexto de educación formal en diferentes niveles y disciplinas, y cuyos géneros son, correspondientemente, llamados géneros académicos. Este tipo de discurso tiene sobre todo propósitos persuasivos, divulgativos y didácticos; expresa credibilidad y prestigio como discurso autorizado; se usa para difundir conocimiento disciplinar, para apoyar la formación de los miembros de una comunidad de especialistas y para mediar las interacciones de esa comunidad (Parodi, 2007).

El discurso profesional, por su parte, es aquel que media en las interacciones del campo laboral, cuyas demarcaciones pueden ser trazadas por las diferentes prácticas de la vida productiva y económica, lo mismo que por las disciplinas que 
subdividen a estas prácticas, las cuales le imprimen una impronta característica y forman tradiciones y hábitos. Este discurso se realiza a través de géneros profesionales tan diversos como el memorando, el proyecto, el oficio, el plan de negocios o el informe técnico, que surgen en respuesta a las necesidades y fines de esas prácticas (Cassany y López-Ferrero, 2010; Navarro, 2012; Navarro y Brown, 2014; Parodi, 2008), y circula entre los integrantes (practicantes) de una misma profesión, y también entre miembros de profesiones distintas y entre la comunidad profesional y un público lego (Parodi, 2007).

Estos dos tipos de discurso se diferencian por sus contextos de uso y circulación, aunque existen puntos de contacto entre ellos: los textos profesionales plasman un conocimiento específico del mundo que fundamenta los propósitos y hábitos de una comunidad profesional, por lo cual es viable entenderlos también como textos académicos; por otra parte, desde un punto de vista interactivo, el discurso de los académicos es profesional porque estos docentes e investigadores son, a su vez, personas que actúan y cumplen fines en un medio laboral.

De acuerdo con Bolívar y Parodi (2015) y Parodi (2007), el discurso académico y el profesional forman parte de un constructo más amplio, una abstracción mayor que los contiene, llamado discurso especializado, que en términos generales se caracteriza por su alto grado de formalidad y su disciplinariedad, en oposición a las interacciones informales y a otras prácticas institucionales que no están relacionadas con el quehacer científico, académico o profesional.

Algunos autores usan los constructos discurso científico (Parodi, 2008) o discurso científico-académico (Navarro y Revel, 2013) para designar al lenguaje y las prácticas de discurso realizadas por los investigadores -los scholars de la tradición anglosajona-, y se deslindan de cualquiera otra forma de discurso que se produzca o circule en el ámbito escolar:

El discurso científico-académico se considera una variedad funcional del lenguaje con rasgos lingüísticos co-ocurrentes propios que circula en textos orales y escritos ligados a organizaciones específicas de las esferas científicas y académicas, y que presenta como objetivo general la producción de conocimiento, especificado a su vez en los objetivos de los géneros discursivos propios (Navarro y Revel, 2013, p. 49).

Su fin es generar y validar conocimientos a través de géneros de investigación (Swales, 2013), entre los que figuran el artículo de investigación, el capítulo de libro, la conferencia y la ponencia (Castelló, 2007). La diferencia principal entre el discurso académico y el científico radica en la prevalencia del andamiaje didáctico con fines educativos que caracteriza al primero, por el cual algunos autores le llaman también discurso pedagógico (Jarpa, 2015).

El criterio de deslinde en este caso es interactivo y epistemológico; existe otro tipo de discurso al interior de la academia que se produce con fines de enseñanza, aprendizaje y evaluación, y que los estudiantes de diferentes niveles tienen que aprender y dominar tan solo para transitar con éxito por el sistema educativo, pero no necesariamente para producir conocimiento nuevo y original (Navarro y Revel, 2013), como veremos más adelante.

Concediendo un valor práctico a estas consideraciones sobre los distintos tipos de discurso, llegamos a una definición amplia de discurso académico que abriga tanto 
"el discurso de los académicos" hecho por investigadores (Bolívar, 2004) como la "lectura y escritura en la universidad", por estudiantes y profesores de licenciatura y posgrado (Bolívar y Beke, 2014), lo mismo que el "lenguaje de la escolaridad" (Schleppegrell, 2004), que asimila al "discurso escolar general" (Parodi, 2007), que se configura por las prácticas de oralidad, lectura y escritura realizadas desde la educación básica hasta la superior.

Para precisar el concepto revisado, proponemos el constructo discurso académico de formación para referirnos a las prácticas de lectura y escritura con propósitos de aprendizaje, los cuales implican "interpretar la información de manera crítica, integrar información obtenida en fuentes diversas y crear información” (Cubo, 2014, p. 17), pero no necesariamente generar nuevos conocimientos. Estas prácticas se efectúan por medio del discurso pedagógico, se cristalizan en géneros académicos de formación que, al cumplir fines de acreditación, se denominan géneros evaluativos (Jarpa, 2015).

Estos últimos son producidos en una situación de asimetría, porque en ella los estudiantes tienen el rol de evaluado (Cubo, 2014) y uno o más docentes cumplen el rol de evaluador. Incluso, si son considerados como géneros académicos de formación (según la denominación de Navarro y Revel, 2013), su propósito intrínseco es acreditar las competencias, habilidades y dominio disciplinar adquirido por los estudiantes. En ellos interactúan un profesor (experto) y unos estudiantes (semilegos) en una situación comunicativa de carácter didáctico-pedagógico dentro de un sistema de actividad académico, donde se emplean con fines educativos (Jarpa, 2015).

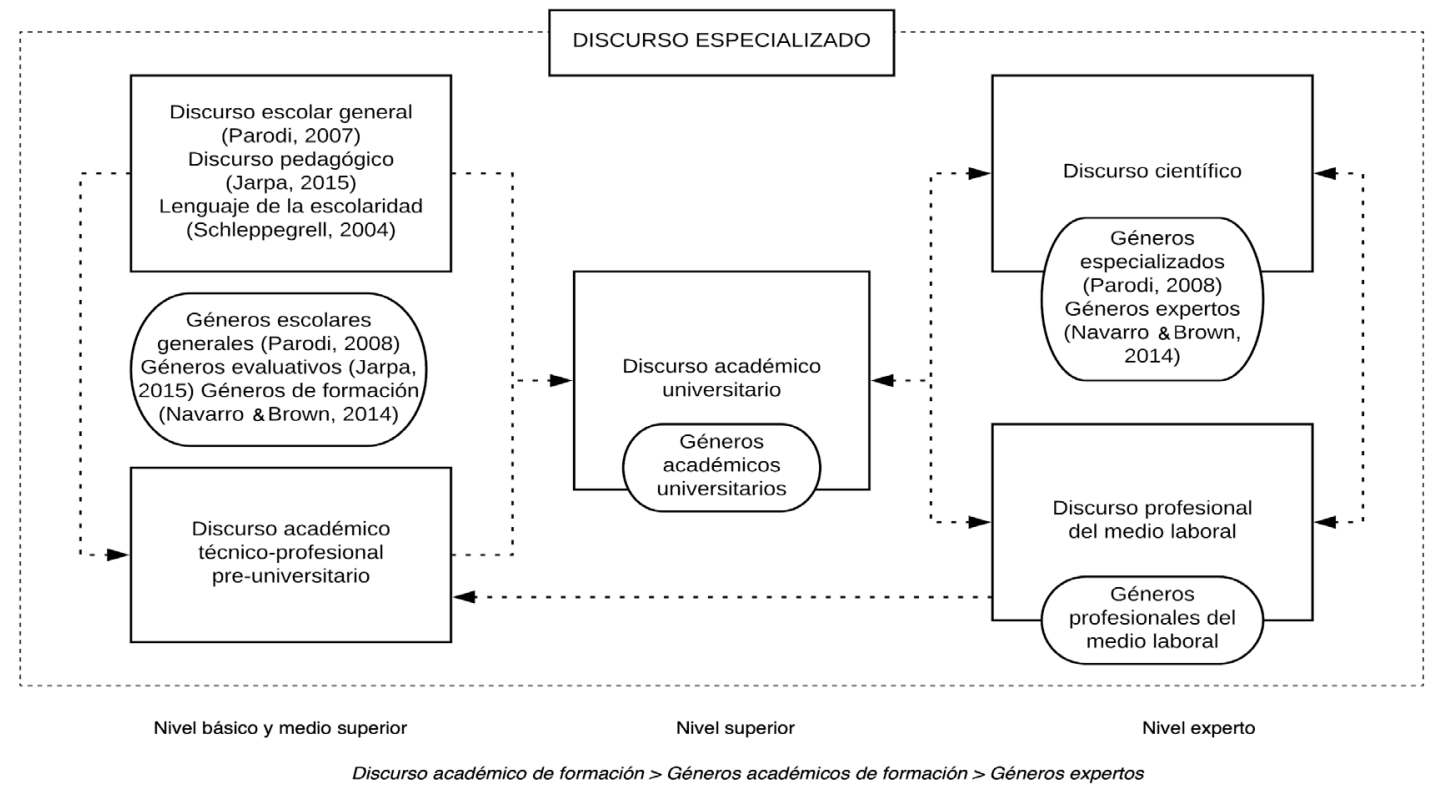

Figura 1. El continuo del discurso especializado y sus géneros. Fuente: Elaboración propia con base en Jarpa (2015), Navarro y Brown (2014), Parodi $(2007,2008)$ y Schleppegrell (2004). 
La figura 1 sintetiza de manera esquemática las comunicaciones y los trayectos entre los contextos que hacen uso del discurso especializado y sus géneros. Estos contextos educativos constituyen sistemas de actividad que desarrollan prácticas letradas usando discursos provenientes del ámbito científico y que habilitan a sus estudiantes en la producción de los textos considerados relevantes por la comunidad, por ser el puente hacia el ejercicio profesional y sus géneros específicos (Navarro, 2012, 2014).

En la citada figura se propone que el discurso especializado es un continuo que va aumentando el nivel de especialización, desde uno menos alto a uno más alto. La columna de la izquierda comprende las prácticas discursivas de la escolaridad básica y media, que, junto con la columna central de la formación universitaria, representan el campo en que se emplean el discurso académico de formación y los géneros académicos de formación, cuyas características ya hemos explicado. A partir de la diversificación disciplinar que opera en la universidad, y a causa de la profundización en los conocimientos que implica la obtención de un grado profesional, podemos distinguir, en la columna de la derecha, un nivel más complejo del discurso especializado: el nivel experto, con sus géneros.

\section{ESCRITURA Y CONOCIMIENTO}

Como hemos explicado, pertenecer a una cultura disciplinar implica "participar de un conjunto de prácticas de lectura y escritura consensuadas" por una comunidad (Navarro y Brown, 2014, p. 67). Una participación exitosa en esa comunidad exige, asimismo, el manejo competente de dichas prácticas. La cuestión del "manejo competente de esas prácticas" (Navarro y Brown, 2014, p. 67) -entre ellas, la escritura académica- como constitutivas de la literacidad escolar y el desempeño previsto en esas prácticas como indicador del logro de aprendizajes constituyen un asunto central que representa la razón práctica de un centro de escritura.

En la figura 1, el gradiente de menor a mayor nivel de especialización muestra una sucesión de eventos letrados que un sujeto en formación debe transitar para llegar a construir un dominio discursivo en los ámbitos académico y profesional. Este recorrido representa un proceso de alfabetización permanente: desde la perspectiva de esa persona en formación, esta progresión es experimentada como un ambiente de inculturación disciplinar a través del cual va enfrentando escenarios y géneros diversos que retan sus habilidades y conocimientos previos (Parodi, 2007, 2008).

En dicho proceso, algunos de los géneros serán leídos solo como medios de acceso a conocimientos específicos (Parodi, 2008); otros constituirán tareas de escritura que persigan el propósito de comunicar informaciones también específicas, pero algunos de ellos serán seleccionados por la comunidad como los que el aprendiz debe dominar; se trata de géneros "puente" a los géneros expertos (Navarro, 2012) que cumplen funciones relevantes en las prácticas sociales de esa comunidad.

En este mismo marco de ideas, y dado que el tema de interés de este artículo es sustentar los conceptos que fundamentan la labor de un centro de escritura, es necesario considerar las exigencias que plantea el discurso y el texto académico en el 
nivel superior para comprender los desafíos que presentan a los estudiantes al estar sujetos a un proceso de alfabetización disciplinar, en especial en lo que se refiere a las prácticas de escritura.

\section{LOS GÉNEROS COMO ACCESO AL CONOCIMIENTO Y EVIDENCIA FORMATIVA}

En primer lugar, hay que notar que en todos los niveles educativos circulan discursos con algún grado de especialización, incluso en los libros de texto, manuales y exámenes de la educación básica y media, con especial interés en la educación superior. Es un hecho que ese tipo de artefactos didácticos fueron escritos por expertos de diversas disciplinas para conseguir propósitos pedagógicos en sus usuarios, que son los aprendices. Esos textos son, en este sentido y contexto, "iniciáticos" que apoyan los fines de la escolarización (Cubo, 2014).

En segundo lugar, es preciso tener presente que entre los fines de las instituciones educativas está, justamente, ofrecer a sus miembros oportunidades de aprendizaje (y, por tanto, experiencia) en esta sucesión progresiva de eventos de inculturación disciplinar y profesional (con matices en los distintos niveles); por ello, en estos contextos cobran particular importancia las nociones de construcción de conocimientos y evaluación. Si tomamos en cuenta esto, resulta muy útil pensar en la existencia de unos géneros académicos de formación que conducen y sostienen el trayecto de los estudiantes hacia el dominio de una selección de géneros expertos, o, por lo menos, de unos géneros evaluativos que acreditarán su competencia a criterio de sus profesores.

Al estar inmersos en un ambiente institucional que valora la adquisición de prácticas epistémicas (como regular, dirigir, explicar, analizar y valorar el pensamiento) dentro de un determinado marco disciplinar, los estudiantes asimilan una cultura académica, es decir, modos de socializar el conocimiento con sus pares y con sus profesores que van creando en ellos modelos de pensamiento $\mathrm{y}$, de manera concomitante, esa inculturación también modela el uso de un lenguaje adecuado a esas formas de razonamiento (Navarro y Revel, 2013).

Al respecto, Russell (1991) puntualiza que en un sistema de educación masiva ni la academia es una comunidad unificada (sino más bien una colección de comunidades, de disciplinas profesionales que compiten, cada una con su propio discurso especializado) ni los niveles educativos son un bloque homogéneo. De este modo, cada tipo de institución desarrolla, en palabras de este autor, "su propia literacidad", sus propias expectativas, generalmente tácitas, de cómo deberían escribir los miembros de esa comunidad y sus estudiantes.

Así pues, se considera que en el contexto de la escolaridad se da una literacidad particular, pero que también al interior de ella hay múltiples literacidades, no solo en función de la diversidad de soportes y planos de lo escrito, que por sí mismos movilizan habilidades diversas para su manejo eficiente, sino porque cada nivel educativo, cada modalidad escolar y cada disciplina profesional alientan la multiplicidad de recursos cognitivos, lingüísticos y retóricos que deben disponer los miembros de una comunidad académica para comunicarse y conseguir sus metas. 


\section{EL TEXTO ACADÉMICO COMO ARTEFACTO LINGÜíSTICO}

Dado que este artículo se enfoca a la revisión de conceptos atinentes a la escritura en el ámbito de la formación inicial de profesores, quienes en su calidad de estudiantes de nivel superior han de dar cuenta de sus aprendizajes por medio de textos, nuestro interés es saber cuáles son las características lingüísticas esperables del texto académico que pudieran representar retos de consideración para los estudiantes al enfrentar tareas de escritura. Para ello, es necesario recuperar algunos aspectos de la relación discurso-género-texto que se han expuesto en los apartados previos.

Como hemos explicado, los textos son la realización concreta de unos géneros que, a su vez, configuran una entidad abstracta que es un determinado tipo de discurso. Del mismo modo, se sabe que los géneros son constructos en los que interactúan una dimensión social, una cognitiva (epistémica) y una lingüística, que las enlaza y las vehicula (Parodi, 2008); por lo tanto, los textos que dan realidad a esos géneros no deben verse como meras "constantes estructurales" o "patrones textuales" que un estudiante ha de aprender a llenar.

En su manifestación concreta, los géneros son variedades de una lengua que operan a través de las tramas de un texto, y que se circunscriben lingüísticamente en virtud de propósitos comunicativos, participantes implicados (escritores y comprendedores), contextos de producción, ámbitos de uso, formas de organización discursiva, soportes y medios, entre otros (Parodi, 2008).

Estos criterios sociocognitivos y lingüísticos sitúan el discurso académico como una variedad funcional específica dentro de una lengua natural (Navarro y Brown, 2014), que se actualiza en un gradiente de textos de menor a mayor especialización, en función de la mayor complejidad de los significados que construyen para cumplir fines específicos (Parodi, 2008). En el entendido de que el texto académico cristaliza ciertas organizaciones retóricas por medio de recursos lingüísticos específicos, aprender a escribirlo implica tener conciencia de estos recursos y aprender a controlarlos para, de ese modo, "poder formar parte de la disciplina con aportes propios" (Navarro y Brown, 2014, p. 77).

Hecha esta contextualización, es conveniente asentar algunos supuestos de partida, mencionados en los apartados anteriores: primero, que la escritura es el medio preferente a través del cual se crea, fija y transmite el conocimiento disciplinar que configura un discurso especializado, cristalizado en géneros prototípicos que se efectúan, a su vez, en textos concretos (Parodi, 2008); segundo, que no obstante la preeminencia de la escritura, todo texto es un artefacto multisemiótico que permite la construcción y el uso de esos conocimientos avanzados por medios diversos (Cassany, 2012; Lemke, 1998; Parodi, 2010); tercero, que como tal, el texto puede ser enfocado meramente como objeto lingüístico, o bien, como un artefacto semiótico implicado en eventos y prácticas sociales (Bolívar, 2007); y cuarto, que los textos funcionan como accesos al conocimiento, pero también a las prácticas discursivas de una comunidad; por ello, aunque la comprensión de los textos es fundamental, solo la producción escrita efectiva de los géneros requeridos por una comunidad particular puede revelar el máximo nivel de competencia discursiva de un sujeto experto en la disciplina (Parodi, 2008). 
Así, el texto académico se conceptualiza como un artefacto lingüístico que media en eventos y prácticas letradas en el ámbito académico, aunque se acepta la co-participación de la imagen, la tipografía, la nomenclatura científica, la notación matemática, los esquemas, los enlaces activos a las redes y cualquier otro sistema simbólico en la construcción del discurso escrito, de acuerdo con las perspectivas de la multimodalidad y las multiliteracidades (Anstey \& Bull, 2018; Cassany, 2012; Lemke, 1998); de igual manera, se acepta que el contexto escolar no es el único en que suceden prácticas letradas y, por tanto, el texto cumple múltiples funciones sociales fuera de la academia.

\section{Características discursivas del texto académico}

Una vez situado el carácter instrumental del texto en el campo de interés de un centro de escritura, en seguida abordamos sus características discursivas y los recursos lingüísticos que se emplean para realizarlas.

Un principio de estilo de la escritura académica es que todo lo que el lector necesita saber ha de estar explícito en el texto; por ello, las ideas toman la posición central y la personalidad del escritor se desplaza al fondo, de modo que las afirmaciones y argumentos sean aceptados por el lector a causa de la lógica o la evidencia, y no por quien escribe. El esfuerzo por mantener a la persona del escritor en el fondo es observado a través de los medios por los cuales el autor se refiere a sí mismo, por lo general usando la tercera persona, el pronombre se impersonal o el plural, pues este distanciamiento aporta credibilidad al discurso (Monippally \& Pawar, 2010).

La formalidad requerida para la escritura académica implica cambiar de registro y evitar las expresiones coloquiales, así como seguir de cerca las convenciones de los géneros. Una de las convenciones más destacadas es la forma de citar las fuentes o los puntos de vista de otros; el grado de rigor con que se hace referencia a las fuentes refleja la intención del escritor del texto académico de lograr la objetividad, y no apoyarse en la opinión o la intuición (Beke, 2011).

Otros rasgos de la escritura académica son el tono tentativo, más matizado que categórico, que se observa en recursos atenuadores; también, la claridad y la precisión, que se materializan por medio de las elecciones léxicas y sintácticas; por ello, el lenguaje tiende a ser especializado, y las oraciones y los párrafos son más largos y complejos que en otros tipos de escritura (Monippally \& Pawar, 2010).

Para evitar ambigüedades e interpretaciones erróneas, y lograr la claridad en el mensaje, en los textos académicos se emplean organizadores textuales que guían al lector. Por un principio de economía e informatividad, se utilizan sustantivos y frases nominales que concentren el máximo de información, por lo que hay un elevado número de nominalizaciones, más que en otros discursos no académicos (Cademártori, Parodi y Venegas, 2006; Parodi, 2007), y una mayor densidad léxica. Para evitar la redundancia, en los textos académicos se cuida que no haya repeticiones, y se da paso a un vocabulario diverso; por precisión, se eliminan los adjetivos vacíos; la sintaxis se controla para mantenerla en su orden habitual o canónico, y se recurre a abundantes expresiones formulaicas y metalenguaje (Hyland \& Tse, 2004). 


\section{Características lingüísticas del texto académico}

En los textos académicos co-ocurren de manera sistemática rasgos lingüísticos particulares en torno a temáticas específicas no cotidianas que exigen experiencia previa. La función del lenguaje que predomina en ellos es la comunicativa referencial, y uno de sus rasgos prototípicos es el empleo de un léxico especializado. Se caracterizan por su alto grado de generalización y abstracción semántica; son textos altamente elaborados que utilizan un registro formal de la lengua y procuran ser objetivos (Parodi, 2007).

Algunos de los recursos lingüísticos utilizados para organizar el conocimiento disciplinar en los textos académicos son el encapsulamiento de hipótesis o afirmaciones en etiquetas nominales, y el empleo, como hemos mencionado, de un léxico especializado (Halliday \& Martin, 1993; Navarro y Revel, 2013). Además, ya se ha advertido que en algunas disciplinas los textos presentan información no verbal por medio de lenguaje matemático y nomenclaturas de alto grado de especialización, como tablas, figuras y diagramas (Parodi, 2007).

En cuanto al uso del lenguaje, es pertinente señalar que el léxico es, junto con la morfosintaxis, uno de los principales niveles o componentes del análisis lingüístico (Alcaraz y Martínez, 1997). "El léxico en una lengua es el repertorio de palabras establecidas que los hablantes pueden usar cuando hablan y a las que pueden recurrir para entender lo que oyen" (Clark, 1995, p. 2); aunque en principio los términos léxico y vocabulario pueden ser intercambiables, se suele usar léxico para nombrar la clase abierta de todas las palabras portadoras de significado disponibles en una lengua, mientras que vocabulario alude a una clase cerrada, una reducción del léxico para fines de análisis. Desde un enfoque paradigmático, en este trabajo aludimos al léxico empleado por los estudiantes en sus textos como la serie de elecciones realizadas por ellos en la construcción del discurso a partir del sistema, y no a la clase cerrada del vocabulario específico de cada texto.

En particular, el tipo de léxico que se espera en la escritura académica es especializado, técnico, denso; también se confía en que las entidades o sujetos sean nombrados explícitamente (es decir, que los participantes del discurso sean lexicalizados; no asumidos), y que haya abundantes nominalizaciones (Schleppegrell, 2001). Asimismo, en este tipo de textos hay una mayor diversidad y densidad léxica respecto a interacciones informales (Chafe \& Danielewicz, 1987; Snow \& Uccelli, 2009), y también el uso de expresiones prestigiosas, propias de la comunicación formal, alejada de coloquialismos. Del escritor académico, se espera que sus elecciones léxicas sean precisas, a diferencia de la ambigüedad que caracteriza al habla y la escritura coloquial; que elija emplear tanto términos técnicos como conceptos abstractos, en vez de los conceptos del sentido común y el discurso no escolar (Snow \& Uccelli, 2009).

En cuanto al tipo de elecciones léxicas, Halliday y Martin (1993) destacan el recurso de la nominalización para condensar información en el discurso académico, de ahí que la vasta mayoría de los términos técnicos son sustantivos, muchos de ellos resultan de la abstracción de procesos verbales (por ejemplo, lixiviación, precipitación, calcificación, inflación, descripción, narración). Estos procesos se abstraen para usarlos como principios explicativos y clasificatorios en las diferentes disciplinas (por ejemplo, el término erosión condensa y clasifica una serie de procesos 
que tendrían que explicarse con una serie de pasos, los cuales, a su vez, condensan procesos previos y efectos posteriores: deforestación, abrasión, sedimentación, desertificación).

Diversos autores han evidenciado la complejidad de los procesos de abstracción que subyacen a la interpretación y producción de textos académicos (Bello, 2016; Fang, Schleppegrell \& Cox, 2006; García, Hall y Marín, 2005). Esta complejidad tiene su origen en los distintos valores y funciones que cobran principalmente, pero no de modo exclusivo, los sustantivos, las frases nominales y las nominalizaciones en el tejido del discurso: no solo nombran sus entidades y participantes, sino que construyen significados de alto grado de especialización disciplinar, condensan información y cohesionan segmentos textuales de manera anafórica y catafórica, además de aportar una carga modal, atenuante o evaluativa a ciertas palabras y frases, y forman un metalenguaje característico de este discurso (Jiang \& Hyland, 2015, 2016; Hyland \& Tse, 2004).

A la vez, la dificultad de reconocer, emplear y monitorear estos recursos con funcionalidades múltiples se relaciona con los diferentes tratamientos didácticos que se le dan al lenguaje en las materias escolares en los distintos niveles educativos. Halliday y Martin (1993) sostienen que, mientras en unos casos la tradición de enseñanza de alguna disciplina facilita la adopción de términos como si fueran requisitos, en otros se pierde de vista la utilidad del término para construir todo un concepto complejo.

La importancia de destacar el empleo de términos técnicos como una parte esencial de toda escritura técnica y científica radica en el hecho de que cada campo del conocimiento y cada especialidad emplean típicamente un determinado léxico que transmite una variedad de conceptos especializados que usan lenguaje técnico. Estos términos especiales comunican significados concentrados que han sido construidos a lo largo de periodos significativos de estudio en un campo, y su valor yace en la forma en que cada término condensa una masa de información en una sola palabra (Perelman, Paradis \& Barret, 1998).

El carácter técnico o especializado de una palabra es un aspecto funcional de ella, lo cual supone que en un contexto una palabra tiene la función de condensar significados de un dominio especializado -y, por lo tanto, entra a formar parte del sistema de ese campo de conocimiento-, mientras que en otros puede cumplir funciones diferentes. La estrecha relación del léxico técnico y especializado con las disciplinas y sus materias se refleja en las distintas proporciones de este tipo de léxico que se han encontrado en corpus de diversas especialidades (Chung \& Nation, 2004; Coxhead, 2017).

Para Halliday y Martin (1993), la importancia de la terminología técnica en la escritura académica y científica radica en que, gracias al uso de léxico especializado, se pueden realizar taxonomías del mundo, de sus fenómenos y entes, mucho más precisas y comunicables que las clasificaciones del sentido común, basadas en lo que puede ser observado directamente con los sentidos. La escritura técnica está asociada a las disciplinas o los campos de la ciencia de manera estrecha, y la ciencia se centra en entender cómo está organizado el mundo y cómo ha llegado a ser así, lo cual se comunica a través de explicaciones y desarrollo de 
procesos. Ambos modos del discurso requieren terminología especializada del campo para interpretar y comunicar con eficiencia esas taxonomías y procesos; esto implica dominar los crecientes grados de abstracción que particularizan el discurso académico.

Coxhead (2017), finalmente, hace hincapié en el reto que constituye el aprendizaje del léxico técnico especializado. Señala que ser capaz de expresar por escrito conceptos académicos con precisión y emplear un léxico apropiado y con fluidez es una prueba de la pertenencia a una comunidad académica. Sin embargo, por lo regular, los estudiantes no adquieren este tipo de léxico fuera del ámbito académico y es posible que no lo aprendan solo mediante la lectura.

En resumen, el léxico que se emplea en la escritura de textos académicos se caracteriza por ser más variado, más informativamente denso y preciso en relación con otros usos del lenguaje coloquiales e informales, y por constituir un vocabulario especializado, de contenido disciplinar y más abstracto que el usado por los estudiantes en sus interacciones cotidianas. Por ello, el empleo de un léxico con estas características puede ser considerado un indicador de dominio de la escritura académica.

En la figura 2 resumimos las características del lenguaje esperables en la escritura de textos académicos, que contrastan con el lenguaje coloquial informal al que tienen mayor acceso los estudiantes.

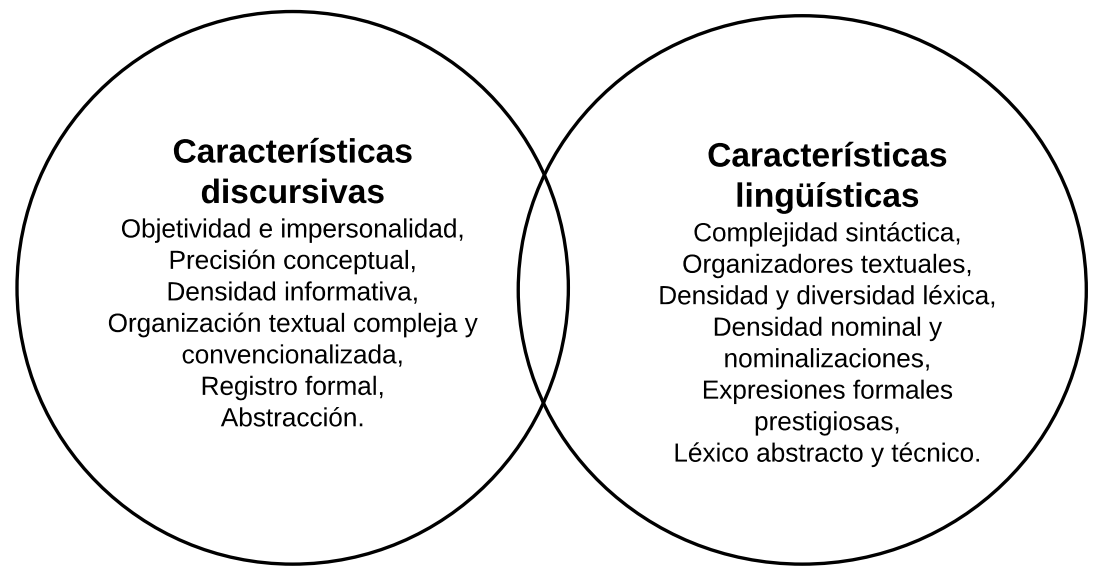

Figura 2. Características del texto académico. Fuente: Elaboración propia con base en Halliday y Martin (1993), Hyland y Tse (2004), Schleppegrell (2001), Snow y Ucelli (2009) y Zwiers (2008).

Con todo lo mencionado, se entiende cómo "el contexto predice el texto y viceversa”, en una serie de imbricaciones entre los contenidos ideacionales, ideológicos, el género, el registro y los recursos léxico-gramaticales que sirven al hablante para construir significados en el ámbito académico (Halliday, 2007; Halliday \& Martin, 1993; Motta-Roth, 2009).

\section{CONCLUSIONES}

Con base en las consideraciones vertidas en este artículo, podemos afirmar que el conocimiento del discurso y el lenguaje que se cristaliza en los textos académicos en el nivel superior puede llevar a un análisis situado en la formación inicial de 
profesores; este análisis permitiría vislumbrar los retos a los que se enfrentan los estudiantes durante su inserción en las prácticas académicas valoradas por la institución educativa, al interior del proceso de inculturación que tiene un carácter social, cognoscitivo y lingüístico.

Una de las prácticas a las que mayor valor epistémico se le confiere en el contexto de la educación es la escritura de textos que respondan a las expectativas de la comunidad académica, y entre esas expectativas se encuentra el manejo de un discurso especializado, abstracto, objetivo, organizado y complejo en la sintaxis y el léxico, diferente al que los estudiantes emplean en su vida cotidiana. La caracterización de los géneros propios de la formación y el desarrollo profesional de los docentes es un paso necesario para conocer mejor esas prácticas en el ámbito de las escuelas normales.

Para realizar su labor, un centro de escritura asume que el dominio de la escritura académica está vinculado a la apropiación y al uso en la escritura misma de unos recursos lingüísticos que configuran los rasgos característicos de los géneros académicos, o al menos de aquellos que gozan de mayor prestigio como vehículos de comunicación de significados disciplinares. El manejo eficiente de esos recursos lingüísticos relevantes en el texto académico demuestra ante los miembros expertos de una comunidad académica que el estudiante ha adquirido unas habilidades y unos conocimientos que le permiten participar con efectividad en las prácticas discursivas que sostienen y dan sentido a esa comunidad.

De este modo, los textos producidos en el ámbito escolar se presentan al interesado en sostener procesos didácticos, de evaluación, asesoría y acompañamiento de la escritura académica, como artefactos lingüísticos que median la participación de los estudiantes en las prácticas letradas escolares. A la vez, los textos son un reflejo de la literacidad alcanzada por ellos, pues, como señalamos, aunque la comprensión de textos académicos es importante para los procesos de escolarización e inculturación disciplinar, es la capacidad de escribirlos de modo adecuado la que se considera el rito de paso a la academia, a la disciplina y, más adelante, al mundo laboral. Una exploración desde varios flancos (lingüístico-discursiva, pragmática, sociocognitiva, interaccional) en los instrumentos de inculturación usuales en la formación de docentes daría materia para elaborar un "perfil letrado" -una literacidad- suficiente, deseable o necesaria para que se lleven a cabo esos procesos.

En síntesis, la literacidad es una competencia compleja de la mayor relevancia porque sus alcances facilitan o dificultan los procesos de inculturación disciplinar; esta se sostiene en la competencia lingüística de los estudiantes, que requieren utilizar el lenguaje no solo para comunicarse oralmente y por escrito, sino para representar, interpretar y comprender la realidad, para construir conocimientos, organizar y regular el pensamiento, las emociones y la conducta. Los proyectos de alfabetización académica tienen como propósito fortalecerla y ampliarla.

De nuestra revisión se desprende que, para emprender una iniciativa de alfabetización académica en cualquier institución escolar, es necesario tomar en consideración el contexto, porque constituye un sistema de actividad cuyas particularidades (se) configuran (con) discursos (Motta-Roth, 2009).

Por ello, en relación con una institución formadora de docentes, es importante caracterizar de manera profunda el conjunto de habilidades de todo tipo que 
componen la literacidad necesaria, deseable o suficiente para la formación inicial de profesores, para su acreditación y su acceso a la vida profesional. Lo mismo se puede decir del tipo de discurso pedagógico (para la formación inicial y continua), académico (para la evaluación de aprendizajes y la acreditación) y profesional (para el acceso al servicio profesional docente) que media en las prácticas de esa comunidad de formación institucional.

Asimismo, se requiere un conocimiento objetivo y contextualizado de los géneros que cristalizan dichos discursos, sus características como superestructuras y macroestructuras retóricas, y también de sus rasgos léxico-gramaticales, como instrumentos de las interacciones de esa comunidad.

De toda esta información, al menos de manera inicial, se puede desprender un proyecto de alfabetización académica que se realice en un centro o programa de escritura, cuyas metas, procedimientos, agentes y mecanismos de seguimiento (evaluación) sean producto del autoconocimiento de la cultura académica de la comunidad e institución en que se inserta.

Agradecimientos

Nuestro agradecimiento a Adriana Bolívar y a Giovanni Parodi, in memoriam.

\section{REFERENCIAS BIBLIOGRÁFICAS}

Alcaraz, E. y Martínez, M. A. (1997). Diccionario de lingüística moderna. Barcelona: Ariel

Álvarez, T. (2010). Competencias básicas en escritura. Barcelona: Octaedro.

Anstey, M. \& Bull, G. (2018). Foundations of multiliteracies. Reading, writing and talking in the 21st Century. Nueva York: Routledge.

Barton, D. \& Hamilton, M. (2010). La littératie: une pratique sociale. Langage et Societé, núm. 3, vol. 133, pp. 45-62. http://dx.doi.org/10.3917/ls.133.0045

Bazerman, Ch. (2005). Reference guide to writing across the curriculum. Indiana: Parlor Press/The WAC Clearinghouse.

Bello, I. (2016). Cognitive implications of nominalizations in the advancement of scientific discourse. International Journal of English Studies, núm. 16, vol. 2, pp. 1-23. http://dx.doi.org/10.6018/ijes/2016/2/262921

Beke, R. (2011). Las voces de los otros en el discurso académico. Caracas: Comisión de Estudios de Postgrado, Facultad de Humanidades y Educación, Universidad Central de Venezuela.

Bolívar, A. (2007). El análisis interaccional del discurso: del texto a la dinámica social. En A. Bolívar (comp.). Análisis del discurso. ¿Por qué y para qué? (pp. 247-277). Caracas Venezuela: Universidad Central de Venezuela/Los Libros de El Nacional.

Bolívar, A. (2004). Análisis crítico del discurso de los académicos. Revista Signos, núm. 37, vol. 55, pp. 1-12. http://dx.doi.org/10.4067/S071809342004005500001

Bolívar, A. y Beke, R. (2014). Lectura y escritura para la investigación. Caracas: Universidad Central de Venezuela. 
Bolívar, A. y Parodi, G. (2015). Academic and professional discourse. En M. Lacorte (ed.). The Routledge Handbook of Hispanic Applied Linguistics (pp. 459475). Nueva York: Routledge.

Cademártori, Y., Parodi, G. y Venegas, R. (2006). El discurso escrito y especializado: caracterización y funciones de las nominalizaciones en los manuales técnicos. Literatura y Lingüística, núm. 17, pp. 243-265. http://dx.doi. org/10.4067/S0716-58112006000100015

Carlino, P. (2012). Escribir, leer y aprender en la universidad. Una introducción a la alfabetización académica. Buenos Aires: Fondo de Cultura Económica.

Carlino, P. (2004). Escribir a través del currículum. Tres modelos para hacerlo en la universidad. Lectura y Vida. Revista Latinoamericana de Lectura, núm. 1, año 25, pp. 16-27. Recuperado de https://media.utp.edu.co/ referencias-bibliograficas/uploads/referencias/articulo/248-escribir-atravs-del-curriculum-tres-modelos-para-hacerlo-en-la-universidadpdfjQyDB-articulo.pdf

Carlino, P. (2003). Alfabetización académica. Un cambio necesario, algunas alternativas posibles. Educere, núm. 20, vol. 6, pp. 409-420. Recuperado de http://erevistas.saber.ula.ve/index.php/educere/article/view/12363

Cassany, D. (2012). En línea. Leer y escribir en la red. Barcelona: Paidós.

Cassany, D. y López-Ferrero, C. (2010). De la universidad al mundo laboral: continuidad y contraste entre las prácticas letradas académicas y profesionales. En G. Parodi (ed.). Alfabetización académica y profesional en el siglo XXI: leer y escribir desde las disciplinas (pp. 347-374). Barcelona: Planeta Ariel.

Castelló, M. (coord.) (2007). Escribir y comunicarse en contextos científicos y académicos: conocimientos y estrategias. Barcelona: Graó.

Chafe, W. L. \& Danielewicz, J. (1987). Properties of spoken and written language. En R. Horowitz y F. J. Samuels (eds.). Comprehending oral and written language (pp. 83-113). Nueva York: Academic Press.

Chung, T. \& Nation, I. S. P. (2004). Identifying technical vocabulary. System, núm. 32, vol. 2, pp. 251-263. https://doi.org/10.1016/j.system.2003.11.008

Clark, E. V. (1995). The lexicon in acquisition. Nueva York: Cambridge University Press.

Coxhead, A. (2017). Approaches and perspectives on teaching vocabulary for discipline-specific academic writing. En J. Flowerdew y T. Costley (eds.). Discipline-specific writing. Theory into practice (pp. 62-76). Nueva York: Routledge.

Cubo, L. (2014). Escritura de formación en la universidad. En F. Navarro (coord.). Manual de escritura para carreras de humanidades (pp. 17-25). Buenos Aires: Editorial de la Facultad de Filosofía y Letras de la Universidad de Buenos Aires.

Fang, Z., Schleppegrell, M. J. \& Cox, B. E. (2006). Understanding the language demands of schooling: Nouns in academic registers. Journal of Literacy Research, vol. 38, núm. 3, pp. 247-273. Recuperado de http://journals.sagepub. com/https://doi.org/10.1207/s15548430jlr3803_1

Galán, R. M. y Ormsby, L. (2011). El centro de escritura como puente en la educación superior: el Centro de Aprendizaje, Redacción y Lenguas del ITAM. En I Congreso Nacional de Expresiones de Cultura Escrita (pp. 173-181). México: ITAM/BUAP/Consejo Puebla de Lectura/IIDE-UABC. 
García, M. M, Hall, B. y Marín, M. (2005). Ambigüedad, abstracción y polifonía del discurso académico: interpretación de las nominalizaciones. Revista Signos, vol. 38, núm. 57, pp. 49-60. https://dx.doi.org/10.4067/S071809342005000100004

Halliday, M. A. K. (2013). El lenguaje como semiótica social. La interpretación social del lenguaje y del significado. México: FCE.

Halliday, M. A. K. (2007). Language and education. J. J. Webster (ed.). Nueva York: Continuum.

Halliday, M. A. K. \& Martin, J. (1993). Writing science. Londres: The Falmer Press.

Hyland, K. \& Tse, P. (2004). Metadiscourse in academic writing: A reappraisal. Applied Linguistics, vol. 25, núm. 2, pp. 156-177. https://doi.org/10.1093/ applin/25.2.156

Jarpa, M. (2015). Escritura en las disciplinas: géneros académicos evaluativos en un programa de posgrado de biotecnología. En G. Parodi y G. Burdiles (eds.). Leer y escribir en contextos académicos y profesionales: géneros, corpus y métodos (pp. 221-255). Santiago de Chile: Ariel.

Jiang, F. y Hyland, K. (2016). Nouns and academic interactions: A neglected feature of metadiscourse. Applied Linguistics, vol. 23, pp. 1-25. Oxford University Press. https://doi.org/10.1093/applin/amw023

Jiang, F. \& Hyland, K. (2015)."The fact tha": Stance nouns in disciplinary writing. Discourse Studies, núm. 17, vol. 5, pp. 1-22. Sage Publications. https:// doi.org/10.1177/1461445615590719

Lemke, J. (1998). Multiplying meaning: Visual and verbal semiotics in scientific text. En J. R. Martin y R. Veel (eds.). Reading science (pp. 87-113). Londres: Routledge.

Molina-Natera, V. (2019). Centros y programas de escritura en Latinoamérica: teorías, prácticas y administración. Santiago de Cali: Pontificia Universidad Javeriana, Sello Editorial Javeriano.

Molina-Natera, V. (2016). Los centros de escritura en Latinoamérica: consideraciones para su diseño e implementación. En G. Bañales Faz, M. Castelló Badía y N. A. Vega López (coords.). Enseñar a leer y escribir en la educación superior. Propuestas educativas basadas en la investigación (pp. 341-362). México: Universidad Autónoma de Tamaulipas/Consejo Puebla de Lectura/CEIDEA/ Fundación SM.

Molina-Natera, V. (2015) (ed.). Panorama de los centros y programas de escritura en Latinoamérica. Santiago de Cali: Pontificia Universidad Javeriana, Sello Editorial Javeriano.

Molina-Natera, V. (2014). Centros de escritura: una mirada retrospectiva para entender el presente y futuro de estos programas en el contexto latinoamericano. Legenda, núm. 18, vol. 18, pp. 9-33. Recuperado de http://erevistas. saber.ula.ve/index.php/legenda/article/view/5205

Molina-Natera, V. y López, K. (2019b). Estado de la cuestión de los centros y programas de escritura en Latinoamérica. Revista Colombiana de Educación, núm. 78. https://doi.org/10.17227/rce.num78-8066.

Montes, S. y Vidal, M. (2017). Diseño de un programa de escritura a través del currículum: opciones teóricas y acciones estratégicas. Lenguas Modernas, núm. 50, vol. 73, pp. 73-90. Recuperado de https://lenguasmodernas.uchile. cl/index.php/LM/article/view/49252/53119 
Montoya, S. (2018). Defining literacy. Hamburgo: Unesco/GALM Fifth Meeting.

Motta-Roth, D. (2009). The role of context in academic text production and writing pedagogy. En Ch. Bazerman, A. Bonini \& D. Figueiredo (eds.) (2009). Genre in a changing world (pp. 321-340). West Lafayette, Indiana: The WAC Clearinghouse/Parlor Press. https://doi.org/10.37514/PERB.2009.2324.2.16

Moyano, E. I. (2017). Diseño e implementación de programas de lectura y escritura en el nivel universitario: principios y estrategias. Lenguas Modernas, núm. 50 vol. 73, pp. 47-72. https://lenguasmodernas.uchile.cl/index.php/ LM/article/view/49251/53100

Monippally, M. M. \& Pawar, B. (2010). Academic writing. A guide for management students and researchers. Nueva Delhi: Response.

Navarro, F. (coord.) (2014). Manual de escritura para carreras de humanidades. Buenos Aires: Editorial de la Facultad de Filosofía y Letras de la Universidad de Buenos Aires.

Navarro, F. (2012). ¿Qué son los géneros profesionales? Apuntes teórico-metodológicos para el estudio del discurso profesional. En A. Cristófalo y J. Ledesma (eds.). Actas del IV Congreso Internacional de Letras "Transformaciones culturales. Debates de la teoría, la crítica y la lingüística en el Bicentenario" (Buenos Aires, 22-27 noviembre de 2010). Buenos Aires: Universidad de Buenos Aires. Recuperado de http://eventosacademicos.filo.uba.ar/index.php/CIL/IV-2010/paper/viewFile/2813/1231

Navarro, F. y Brown, A. (2014). Lectura y escritura de géneros académicos. Conceptos básicos. En F. Navarro (coord.). Manual de escritura para carreras de humanidades (pp. 55-100). Buenos Aires: Editorial de la Facultad de Filosofía y Letras de la Universidad de Buenos Aires.

Navarro, F. y Revel, A. (2013). Escribir para aprender. Disciplinas y escritura en la escuela secundaria. Buenos Aires: Paidós.

Parodi, G. (2010). Multisemiosis y lingüística de corpus: artefactos (multi)semióticos en los textos de seis disciplinas en el corpus PUCV-2010. Revista de Lingüística Teórica y Aplicada, núm. 48, vol. 2, pp. 33-70. http://dx.doi. org/10.4067/S0718-09342014000200002

Parodi, G. (2008). Géneros del discurso escrito: hacia una concepción integral desde una perspectiva sociocognitiva. En G. Parodi (ed.). Géneros académicos y géneros profesionales: accesos discursivos para saber y hacer (pp. 17-37). Valparaíso, Chile: Ediciones Universitarias de Valparaíso-Pontificia Universidad Católica de Chile. Recuperado de http://www.euv.cl/archivos_ pdf/generos.pdf

Parodi, G. (2007). El discurso especializado escrito en el ámbito universitario y profesional: constitución de un corpus de estudio. Revista Signos, núm. 40, vol. 63, pp. 147-178. http://dx.doi.org/10.4067/S071809342007000100008

Perelman, L. C., Paradis, J. \& Barret, E. (1998). The Mayfield Handbook of Technical and Scientific Writing. Mountain View, CA: Mayfield Publishing Company.

Russell, D. (1991). Writing in the academic disciplines, 1870-1990. A curricular history. Carbondale, IL: Southern Illinois University Press. Recuperado de https://lib.dr.iastate.edu/engl_books/4

Schleppegrell, M. J. (2004). The language of schooling. A functional linguistics perspective. Nueva Jersey: Lawrence Erlbaum. 
Schleppegrell, M. J. (2001). Linguistic features of the language of schooling. Linguistics and Education, núm. 12, vol. 4, pp. 431-459. Recuperado de http://blogs.ubc.ca/lled3602015/files/2015/08/Schleppegrell_Linguistic_Features.pdf

Snow, C. E. \& Uccelli, P. (2009). The challenge of academic language. En D. R. Olson \& N. Torrance (eds.). The Cambridge Handbook of Literacy (pp. 112-133). Cambridge: Cambridge University Press. Recuperado de http://nrs.harvard. edu/urn-3:HUL.InstRepos:11654980

Street, B. V. (2005). Understanding and defining literacy. Paper commissioned for the EFA Global Monitoring Report 2006, Literacy for Life. Unesco. Recuperado de https://unesdoc.unesco.org/ark:/48223/pf0000146186

Street, B. V. (1995). Social literacies. Critical approaches to literacy in development, ethnography and education. Londres: Longman.

Street, B. V. (1984). Literacy in theory and practice. Cambridge: Cambridge University Press.

Swales, J. (2013). Research genres. Explorations and applications. Nueva York: Cambridge University Press.

Thaiss, C., Bräuer, G., Carlino, P., Ganobcsik-Williams, L. \& Sinha, A. (2012). Writing programs worldwide: Profiles of academic writing in many places. Fort Collins, Colorado: The WAC Clearinghouse/Parlor Press.

Unesco (2014). Alfabetización: una ruta de aprendizaje multimodal para toda la vida. Consideraciones sobre las prácticas de lectura y escritura para el ejercicio ciudadano en un contexto global e intercomunicado. Bogotá: CERLAC-Unesco/ Universidad de Antioquia.

Zavala, V. (2009). La literacidad o lo que la gente hace con la lectura y la escritura. En D. Cassany (comp.). Para ser letrados. Voces y miradas sobre la lectura (pp. 23-35). Barcelona: Paidós.

Zwiers, J. (2008). Building academic language. Essential practices for content classrooms, grades 5-12. San Francisco, CA: Jossey-Bass/IRA. 